\title{
Fine-scale spatial and temporal variations in insecticide resistance in Culex pipiens complex mosquitoes in rural south-eastern Tanzania
}

Nancy S. Matowo 1,2,3,4*, Said Abbasi ${ }^{1}$, Givemore Munhenga 5,6, Marcel Tanner²,3, Salum A. Mapua ${ }^{1}$, David Oullo ${ }^{7}$, Lizette L. Koekemoer ${ }^{5,6}$, Emanuel Kaindoa ${ }^{1,9}$, Halfan S. Ngowo ${ }^{1,8}$, Maureen Coetzee ${ }^{5,6}$, Jürg Utzinger 2,3 and Fredros O. Okumu ${ }^{1,8,9}$

\begin{abstract}
Background: Culex mosquitoes cause considerable biting nuisance and sporadic transmission of arboviral and filarial diseases.

Methods: Using standard World Health Organization procedures, insecticide resistance profiles and underlying mechanisms were investigated during dry and wet seasons of 2015 and 2016 in Culex pipiens complex from three neighbouring administrative wards in Ulanga District, Tanzania. Synergist tests with piperonyl butoxide, diethyl maleate, and triphenyl phosphate, were employed to investigate mechanisms of the observed resistance phenotypes. Proportional biting densities of Culex species, relative to other taxa, were determined from indoor surveillance data collected in 2012, 2013, and 2015.

Results: Insecticide resistance varied significantly between wards and seasons. For example, female mosquitoes in one ward were susceptible to bendiocarb and fenitrothion in the wet season, but resistant during the dry season, while in neighbouring ward, the mosquitoes were fully susceptible to these pesticides in both seasons. Similar variations occurred against bendiocarb, DDT, deltamethrin, and lambda-cyhalothrin. Surprisingly, with the exception of one ward in the wet season, the Culex populations were susceptible to permethrin, commonly used on bednets in the area. No insecticide resistance was observed against the organophosphates, pirimiphos-methyl and malathion, except for one incident of reduced susceptibility in the dry season. Synergist assays revealed possible involvement of monooxygenases, esterases, and glutathione S-transferase in pyrethroid and DDT resistance. Morphology-based identification and molecular assays of adult Culex revealed that $94 \%$ were $C x$. pipiens complex, of which $81 \%$ were $C x$. quinquefasciatus, $2 \%$ Cx. pipiens, and $3 \%$ hybrids. About $14 \%$ of the specimens were non-amplified during molecular identifications. Female adults collected indoors were 100\% Cx. pipiens complex, and constituted $79 \%$ of the overall biting risk.
\end{abstract}

Conclusions: The $C$ x. pipiens complex constituted the greatest biting nuisance inside people's houses, and showed resistance to most public health insecticides possible. Resistance varied at a fine geographical scale, between adjacent wards, and seasons, which warrants some modifications to current insecticide resistance monitoring strategies. Resistance phenotypes are partly mediated by metabolic mechanisms, but require further evaluation through biochemical and molecular techniques. The high densities and resistance in Culex could negatively influence the acceptability of other interventions such as those used against malaria mosquitoes.

\footnotetext{
*Correspondence: nstephen@ihi.or.tz

${ }^{1}$ Environmental Health and Ecological Sciences Department, Ifakara

Health Institute, Ifakara, Tanzania

Full list of author information is available at the end of the article
} 
Keywords: Culex pipiens complex, Fine spatial scale and temporal differences, Insecticide resistance, Metabolic resistance, Tanzania

\section{Background}

Culicine mosquitoes, including Aedes, Mansonia, and members of the Culex pipiens and Cx. univittatus complexes, are common across East Africa [1-4]. Of particular importance is the Cx. pipiens complex, generally referred to as the "house mosquito" [5]. It is not only a major cause of biting annoyance to humans but is also a primary vector of many arboviruses and filarial worms that affect more than 1 billion people globally [6-8]. The diseases of concern include Rift Valley fever, dengue, chikungunya, yellow fever, Sindbis, Wesselsbron, o'nyong-nyong, and West Nile arboviruses, filarial worms causing Bancroftian filariasis [6-8], and avian Plasmodium species [9]. Most of these pathogens are maintained in zoonotic cycles with humans being incidental hosts [10]. Culicines are adapted and dominate human habitats, increasing their risks to act as bridge vectors in transmitting pathogens between humans and animals $[9,11,12]$. In Africa there have been several sporadic outbreaks of arbovirus infections such as Rift Valley fever in Kenya and Tanzania as well as dengue fever [13-15] and chikungunya [16, 17].

The World Health Organization (WHO) Global Vector Control Response strategy recognizes the need to integrate surveillance and control of pathogens transmitted by different vector species [18]. Surveillance and management of insecticide resistance are two crucial components $[18,19]$ for effective decision-making on selection, allocation, and implementation of appropriate integrated vector control interventions.

Current vector control interventions in Africa are primarily designed to target malaria vectors, with limited efforts to control other mosquito-borne disease vectors. This is also true for insecticide resistance monitoring [20]. The current emphasis on malaria vectors has resulted in knowledge gaps on species other than Anopheles and their resistance profiles to common insecticides used in public health [21, 22]. Yet these species contribute to the greatest human-biting densities. Culicine densities are usually high, because of the presence of numerous favourable aquatic breeding sites that include man-made stagnant water bodies (e.g. small multipurpose dams, rice paddies, etc.), waste disposal sites, open pit latrines and septic tanks, and flooded vegetation [3, 23, 24]. Lack of resources in many countries has limited expansion of surveillance to non-malaria vectors including the culicines.
Previous studies showed spatial and temporal dynamics of insecticide resistance in mosquito vector populations, and influence of environmental contamiants such as agricultural pesticide residues, and such information has been used to plan resistance monitoring efforts [25-27]. In Tanzania, however, insecticide resistance monitoring is carried out at district level in selected sentinel sites in regions assumed to represent different eco-epidemiological settings [28]. Reports from these assessments provide essential data for country-level decision making. However, such a simplistic approach is inadequate for understanding insecticide resistance, which often varies geographically at finer scales other than at the unit of the district or country level [29].

Besides, data on insecticides resistance and associated mechanisms in Culex species are also lacking in Tanzania. Synergist assays have been deployed as a quick and simple method to assess metabolic resistance in mosquito vectors [30-33]. Synergists act by enhancing insecticides penetration into the mosquito body and inhibit the metabolic enzymes that would otherwise digest the insecticides, hence partially/fully restoring susceptibility [30-33].

In addition, data on insecticide resistance in male mosquito populations are limited inspite of both males and females being exposed to insecticides during vector control interventions. Male mosquito populations substantially contribute in the reproduction and increasing population density and their response to insecticides is also a crucial component. In addition, novel vector control interventions such as spraying of swarms [34] with insecticides directly target male mosquitoes. This suggests the need to monitor insecticide resistance on a regular basis in male mosquitoes.

We investigated the spatial and seasonal variations in susceptibility to insecticides of $C x$. pipiens complex mosquitoes from rural south-eastern Tanzanian villages where there is a high coverage of long-lasting insecticidal nets (LLINs) [35], and a regular usage of agricultural pesticides (Matowo et al., unpublished data). The main objectives of the study were (i) to fill important knowledge gaps on insecticide resistance and species diversity of Culex mosquitoes in the study area; and (ii) to investigate fine-scale spatial and temporal differences in resistance and resistance mechanism in the Culex species. 


\section{Methods}

\section{Study area}

Three neighbouring wards, i.e. Minepa $\left(8.271^{\circ} \mathrm{S}\right.$, $\left.36.677^{\circ} \mathrm{E}\right)$, Lupiro $\left(8.385^{\circ} \mathrm{S}, 36.670^{\circ} \mathrm{E}\right)$, and Mavimba $\left(8.312^{\circ} \mathrm{S}, 36.677^{\circ} \mathrm{E}\right)$, in Ulanga District, south-eastern Tanzania were selected (Fig. 1). These villages have high coverage of LLINs [35] and high agricultural pesticide use for crop protection (Matowo et al., unpublished data). Minimum and maximum distances between the wards was $\sim 4 \mathrm{~km}$ (Minepa to Mavimba) and $\sim 9 \mathrm{~km}$ (Minepa to Lupiro). All three wards lie at an altitude between $120 \mathrm{~m}$ and $350 \mathrm{~m}$ above mean sea level. Average

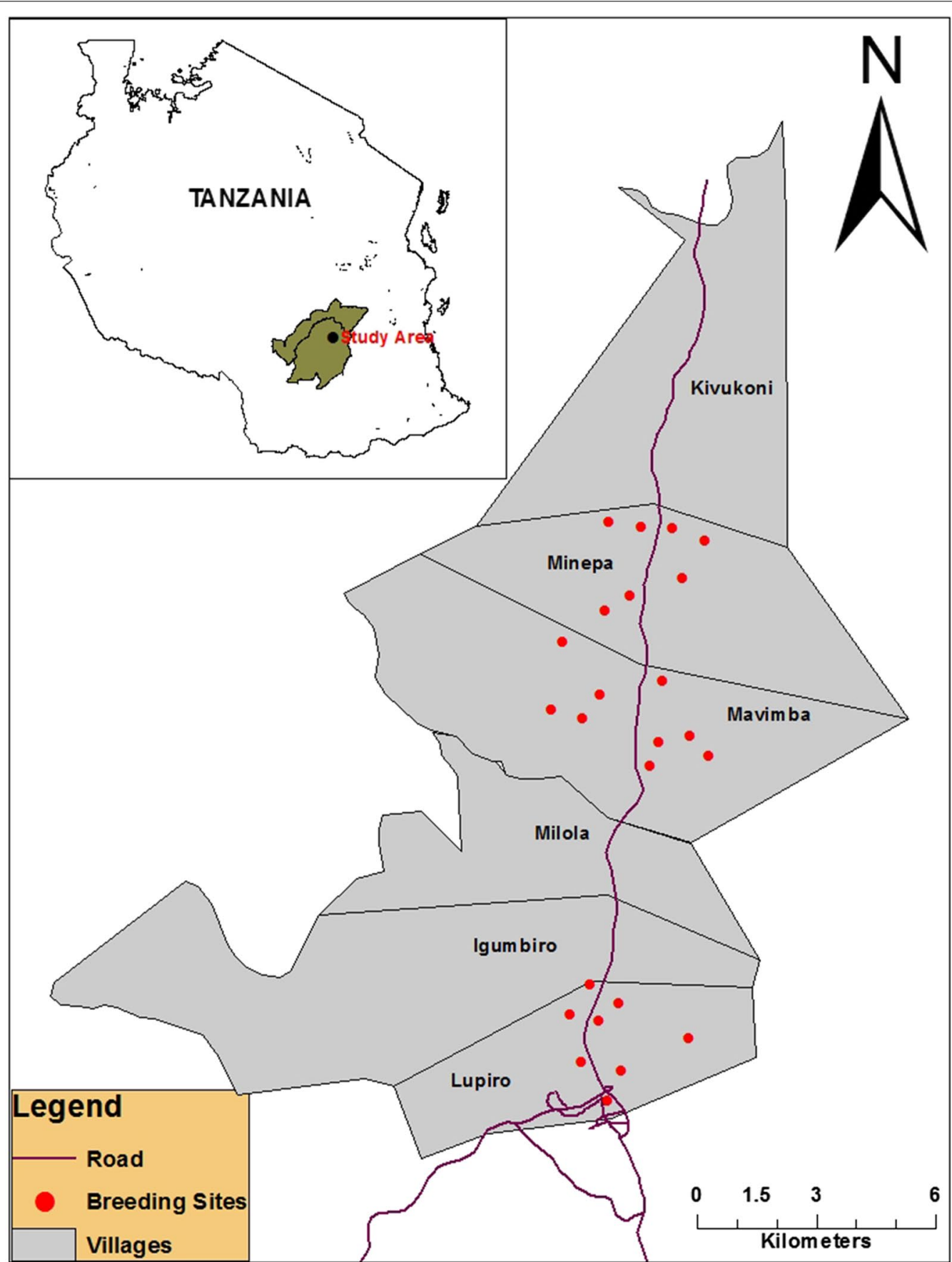

Fig. 1 Locations of mosquito aquatic breeding sites in Minepa, Mavimba, and Lupiro, south-eastern Tanzania, where larvae sampling was conducted between June 2015 and June 2016 
annual precipitation ranges between $1200 \mathrm{~mm}$ and 1800 $\mathrm{mm}$, with the dry season between June and October, a short rainy season in November and December, and the wet season between January and May. Mean daily temperatures over the year vary from $20{ }^{\circ} \mathrm{C}$ to $32{ }^{\circ} \mathrm{C}$, while the relative humidity is $70-90 \%$. Residents practice rice farming [36], which is irrigated during the dry season, so that the area is continuously favourable for mosquito breeding [37]. A national insecticide susceptibility survey in 2011 across 14 districts, including the nearby Kilombero District, reported widespread pyrethroid and DDT resistance in Anopheles mosquitoes [28], but no data on Culex were reported. Recent studies indicated that the two malaria vectors $A n$. arabiensis and $A n$. funestus are highly resistant to pyrethroids, bendiocarb, and DDT, thus compromising vector control efforts [29, 38, 39].

\section{Mosquito sampling and larval rearing}

Mosquito larvae were collected between June 2015 and June 2016, using a standard larval dipping method [40] in three wards, during the dry season (June to December 2015) and wet season (January to May 2016). In each instance, seven to nine randomly selected and georeferenced aquatic habitats were sampled. Larvae were separated into anophelines and culicines to ensure easy adult morphological identification. To assess spatial variations in insecticide resistance, collected larvae were separated per collection site for WHO insecticide resistance assays.

Collected larvae were transferred to the medical entomology laboratory, the "Vector Sphere", at Ifakara Health Institute (IHI; Ifakara, Tanzania), and reared to adults at temperatures of $27 \pm 2{ }^{\circ} \mathrm{C}$ and relative humidity of 70-90\%). Larvae were fed on mud and algae from their original habitats, supplemented with Tetramin fish food (Tetra; Melle, Germany). Emergent adults were separated by sex and taxa, and provided with $10 \%$ glucose solution.

\section{Insecticide susceptibility tests}

Phenotypic insecticide resistance in Culex species in the three study villages was assessed in the dry and wet seasons using standard WHO test kits (Vector Control Research Unit, School of Biological Sciences, Universiti Sains Malaysia, Penang, Malaysia). Adult males and females (3-5 days-old) were exposed in batches of either 20 or 25 individuals according to the discriminating doses of $0.05 \%$ lambda-cyhalothrin, $0.05 \%$ deltamethrin, $0.75 \%$ permethrin, $4 \%$ DDT, $4 \%$ dieldrin, $0.1 \%$ bendiocarb, $0.1 \%$ propoxur, $0.25 \%$ pirimiphos-methyl, and $5 \%$ malathion [41]. The same number of mosquitoes were exposed to oil-impregnated papers as controls. Due to unavailability of reference susceptible Culex mosquitoes, a susceptible colony of $A n$. gambiae (s.s.) (Ifakara strain), was used to validate efficacy of test papers. Knockdown was recorded after 10, 15, 20, 30, 40, 50 and $60 \mathrm{~min}$. After the $60 \mathrm{~min}$ exposure, mosquitoes were transferred to holding tubes and offered $10 \%$ glucose. Final mortality was recorded 24 hours post-exposure [41], after which the mosquitoes were preserved in $1.5 \mathrm{ml}$ Eppendorf tubes containing silica gel for further species identification, using polymerase chain reaction (PCR) assays.

\section{Synergist assays}

Synergist assays were performed using 4\% piperonyl butoxide (PBO), a known inhibitor of monooxygenase, $20 \%$ diethyl maleate (DEM), an inhibitor of glutathione S-transferases (GSTs), and 20\% triphenyl phosphate (TPP), an inhibitor of esterases, as a quick and simple method to assess whether the observed phenotypic resistance had a metabolic enzymes basis [30]. The bioefficacy of synergist papers was tested against a reference laboratory colony (An. funestus) with resistance phenotype mediated by monooxygenases and GSTs [42]. Due to resource limitations, the synergist tests were performed only on female mosquitoes in the dry season in Minepa and Mavimba wards. For each synergist, five cohorts of adults $(n=125)$ were used. The first group was exposed to a synergist (either $4 \%$ PBO, $20 \%$ DEM, or $20 \%$ TPP) for $60 \mathrm{~min}$, and thereafter immediately exposed to WHO test papers impregnated with either $0.75 \%$ permethrin, $0.05 \%$ deltamethrin, $0.05 \%$ lambda-cyhalothrin, or 4\% DDT for another $60 \mathrm{~min}$. The second group was exposed only to the respective WHO test papers, and the third group exposed to the synergist only. Fourth and fifth groups consisted of controls, i.e. filter papers treated with olive oil used to prepare the synergist papers (solvent control), and plain filter papers (environmental control).

\section{Estimating relative densities of Culex mosquitoes and associated biting risk}

The relative proportion of population densities of female Culex species, relative to other mosquito species, was estimated from indoor night collections in 2012, 2013, and 2015 at Minepa, Mavimba, and Kivukoni wards [43, 44], using CDC light traps [45] in 96 randomly selected houses. The mosquitoes were segregated as Anopheles, Culex, Aedes, Mansonia, and other species. The proportion of female Culex population density was used as a proxy for estimating human-biting risk.

\section{Morphological identification of Culex species}

A sub-sample of female Culex mosquitoes $(n=430)$ from the resistance bioassays and female Culex mosquitoes from indoor collections $(n=1053)$ were morphologically identified to determine composition of prevailing species and species complexes using the taxonomic keys of Edwards [46], under a stereo-zoom microscope 
(SZM-LED2, digital Optika ${ }^{\circledR}$ Microscopes; Ponteranica, Italy). To improve identification, the mosquito images were enhanced using OptikalSview software version 3.6.6, and captured using a digital camera (Optika ${ }^{\circledR}$; Ponteranica, Italy) attached to the microscope.

The diagnostic features used for species identification were: (i) presence and number of mesepimeral bristles; (ii) presence or absence of a pale band on proboscis; (iii) presence or absence of white scales on abdomen; (iv) presence or absence of white scales on femur or tibia; and (v) presence or absence of pale-ringed tarsi. To complete the assessment, we first adapted the 1941 morphological keys [46] to focus on just the general diagnostic features and specific features known to occur in Tanzania Culex mosquitoes (Table 1).

\section{Molecular identification of sibling species in the $C x$. pipiens complex}

Morphological identification showed that the Cx. pipiens complex was the most common of all Culex species in the study area. Further molecular identification was conducted using PCR amplification to differentiate two members of the $C x$. pipiens complex (i.e. Cx. pipiens pipiens and $C x$. quinquefasciatus). This PCR targets the acetyl-cholinesterase-2 locus (ace-2). The ace-2 locus was amplified using primers B126, ACEquin, and ACEpip as previously described by Smith \& Fonseca [47].

DNA was extracted from 280 specimens, randomly selected from the morphologically identified $C x$. pipiens complex. A total of $5 \mu \mathrm{l}$ of extracted genomic DNA per sample was amplified in a $20 \mu \mathrm{l}$ reaction mix containing $1 \times$ PCR buffer, $250 \mu \mathrm{M}$ dNTP, $2 \mathrm{mM} \mathrm{MgCl} 2,0.4 \mu \mathrm{M}$ of universal primer and ACEquin, $0.2 \mu \mathrm{M}$ of ACEpip, and 1 unit of Taq DNA polymerase overlaid by a drop of mineral oil. After PCR amplification, $10 \mu \mathrm{l}$ of the DNA fragments were separated by electrophoresis on a $2.5 \%$ agarose gel stained with $0.5 \mu \mathrm{g} / \mathrm{ml}$ ethidium bromide and compared against a 100-bp DNA marker included in the gel. Separated DNA fragments were photographed under ultraviolet light using Kodak Gel Logic 100 imaging system and scored as $C x$. pipiens pipiens (610 bp) or $C x$. quinquefasciatus (274 bp).

\section{Statistical analysis}

The data on susceptibility to insecticides were interpreted following the WHO thresholds established in 2016 [41], where: (i) mean mortality ranging between $98 \%$ and $100 \%$ indicates susceptibility; (ii) mean mortality between $90 \%$ and $97 \%$ indicate possible resistance or presence of resistant genes in the vector populations, but requiring confirmation by repeat bioassays or by a molecular assay; and (iii) mean mortality less than $90 \%$, indicates confirmation of resistance in the test populations. Percentage mean mortality for controls were also calculated, and any tests with mortality greater than $5 \%$, but less than $20 \%$, were corrected using Abbott's formula [48]. Further analysis was done using $\mathrm{R}$ statistical software version 3.0 [49]. Mean mortalities in mosquitoes collected either

Table 1 Identification keys showing main morphological features to distinguish among female Culex collected in three rural wards (Minepa, Mavimba, and Lupiro) in Ulanga District, south-eastern Tanzania. Adapted from the morphological keys by Edwards [46]

\begin{tabular}{|c|c|}
\hline Taxon & Main morphological features for identification of Culex spp. \\
\hline Culexpipiens complex & $\begin{array}{l}\text { Generally smaller size compared to other Culex species } \\
\text { Abdominal tergite with pale basal bands, sternite pale and not banded } \\
\text { Proboscis without a well-defined ring in the middle but pale beneath } \\
\text { Legs and tarsi mostly or entirely dark but hind tibia with a small pale spot at tip } \\
\text { Presence of one lower mesepimeral bristle } \\
\text { Halters yellowish }\end{array}$ \\
\hline Culex (Lutzia) tigripes & $\begin{array}{l}\text { One of the largest Culex species } \\
\text { About } 10 \text { small prominent pale spots on a dark ground marking on femora and tibiae } \\
\text { Abdominal bands, } 6 \text { and } 7 \text { broad, sometimes occupying almost half of the tergites; all sternites pale-scaled, un-banded } \\
\text { Mainly dark proboscis } \\
\text { Dark-scaled wings } \\
3-10 \text { bristles on lower half of the mesepimeron in a more or less regular row }\end{array}$ \\
\hline Culex (Culex) poicilipes & $\begin{array}{l}\text { Sharply-defined median pale yellowish ring on proboscis } \\
\text { Presence of } 7-10 \text { distinct small pale spots on anterior surfaces of front femora and tibiae } \\
\text { Tarsi with pale rings at joints, which are scarcely longer than wide; on joint 4-5 of hind tarsi, pale ring scarcely noticeable } \\
\text { No post-spiracular or pre-alar scales } \\
\text { Wings with all dark scales }\end{array}$ \\
\hline Culex (Culex) duttoni & $\begin{array}{l}\text { Distinctly pale rings on the tarsi and indefinitely ringed proboscis but with whitish scales on the palp almost half } \\
\text { Middle tibia with narrow pale anterior stripe } \\
\text { Presence of } 2-4 \text { lower mesepimeral bristles } \\
\text { Presence of few post-spiracular scales } \\
\text { Dark thorax with no pale scales } \\
\text { Head with pale scales }\end{array}$ \\
\hline
\end{tabular}


in the dry or wet season were compared using $t$-test and any differences considered statistically significant at $P<0.05$. In the synergist tests, the observed 24 hours post-exposure was compared between synergised and un-synergised exposures using $t$-test and any differences considered statistically significant at $P<0.05$. The proportion of female Culex mosquito population density relative to other mosquito species were summarised in a tabular format and used as a proxy for estimating exposure of human to bites.

\section{Results}

\section{Morphological identification of Culex mosquitoes}

A sub-sample of 430 specimens reared from larvae from the three wards (Table 2) were identified as belonging to four Culex species or species complexes as follows: 94\% $(n=405) C x$. pipiens complex; $\%(n=8) C x$. (Lutzia) tigripes; $1 \%(n=3) C x$. (Culex) poicilipes; and $3 \%$ $(n=14)$ Cx. (Culex) duttoni. The 1053 Culex mosquitoes sub-sampled from indoor collections were also identified as members of the $C x$. pipiens complex. Given the dominance of $C x$. pipiens complex, results of insecticide resistance tests are considered most representative of this species complex.

\section{Molecular identifications}

About $94 \%$ of Culex belonged to the Cx. pipiens complex, of which $81 \%$ were verified by PCR as $C x$. quinquefasciatus, $2 \%$ as $C x$. pipiens pipiens, and $3 \%$ as hybrids of $C x$. pipiens pipiens and $C x$. quinquefasciatus. A small proportion of samples (14\%) did not amplify.

\section{Insecticides resistance status of Culex mosquitoes in different wards and seasons}

Table 3 summarizes results for standard WHO susceptibility tests [32] on adult male and female Culex in the three study wards. The reference colony (An. gambiae (s.s.)) used to test insecticidal activity of the test papers

Table 2 Number of adult Culex of different species or species complexes identified from sub-samples emerged from larvae collected in three study wards in Ulanga District, Tanzania, in 2015 and 2016

\begin{tabular}{lllll}
\hline Species & \multicolumn{4}{l}{ Study wards } \\
\cline { 2 - 5 } & Minepa ward & Mavimba ward & Lupiro ward & Total \\
\hline Cx. pipiens complex & 160 & 112 & 133 & 405 \\
Cx. (Lutzia) tigripes & 4 & 1 & 3 & 8 \\
CX. (Culex) poicilipes & 1 & 2 & 0 & 3 \\
CX. (Culex) duttoni & 11 & 0 & 3 & 14 \\
Total & 176 & 115 & 139 & 430 \\
\hline
\end{tabular}

was fully susceptible (100\%) to all candidate insecticides. No mortality was observed upon exposure of wild-caught Culex to untreated papers. The Culex mosquitoes sampled displayed differences in resistance to each insecticide by ward, time of year (dry or wet season), sex (male or female mosquitoes), and insecticides tested.

Overall, lower mortality was observed in the Minepa ward than the other two wards, and females had lower mortalities than males. In addition, resistance to bendiocarb, deltamethrin, lambda-cyhalothrin, and DDT, was higher in the dry season than in the wet season. There was complete resistance or reduced susceptibility to the pyrethroids, except permethrin, against which the mosquitoes (both males and females) from the Minepa and Mavimba wards were fully susceptible regardless of the season. In the Lupiro ward, however, Culex were susceptible to permethrin in the dry season, but resistant to it during the wet season. In the Minepa ward, both male and female Culex were resistant to bendiocarb in the dry season, but fully susceptible in the wet season. Those Culex collected from the Mavimba and Lupiro wards remained fully susceptible to bendiocarb during both seasons. Similar spatio-temporal variations in resistance profiles were observed for male Culex exposed to deltamethrin, lambda-cyhalothrin, and bendiocarb.

\section{Effects of synergists on pyrethroid and DDT resistance phenotypes}

Results of synergist tests on the different resistance phenotypes are detailed in Tables 4 and 5. In the Minepa ward, samples synergized with $4 \%$ PBO exhibited mean mortality of $57.5 \%$ on exposure to $0.05 \%$ lambda-cyhalothrin, compared to $35.0 \%$ in un-synergized cohorts. The difference in mean mortality was marginal, when examined using two-sample $t$-test $\left(t_{(6)}=2.50, P=0.047\right)$. Conversely, synergizing the same population with $20 \%$ TPP did not change the mortality after exposure to lambdacyhalothrin $\left(t_{(6)}=0.23, P=0.827\right)$. Resistant phenotype pre-exposure to $20 \%$ TPP followed by exposure to deltamethrin, resulted in 1.6-fold increase in mortality, relative to exposure to deltamethrin alone (81.3 vs 51.3\%). This difference was statistically significant $\left(t_{(3)}=2.84\right.$, $P=0.030)$. Similarly, there was a statistically significant difference in mortalities after exposure to $0.05 \%$ deltamethrin with or without pre-exposure to $4 \% \mathrm{PBO}$ (93.8 vs $\left.73.8 \% ; t_{(4)}=2.99, P=0.042\right)$. However, there was no difference in mortalities in mosquitoes exposed to $4 \%$ DDT (90\%) with or without pre-exposure to 20\% DEM (95.0 vs $\left.90.0 \% ; t_{(6)}=-1.73, P=0.134\right)$.

In the Mavimba ward, we observed a significantly higher mortality when mosquitoes were pre-exposed to $20 \%$ TPP, followed by lambda-cyhalothrin, as opposed to exposure to lambda-cyhalothrin alone (83.8 vs $72.5 \%$; 
Table 3 Fine-scale spatial and seasonal variations in insecticide susceptibility of Culex mosquitoes collected in three neighbouring wards in the Ulanga District, Tanzania, in the dry season (June-December 2015) and wet season (January-May 2016). Adult mosquitoes exposed for each insecticide were either 20 or 25 per replicate. Results expressed as \% mean mortality 24 hours postexposure

\begin{tabular}{|c|c|c|c|c|c|c|c|}
\hline & \multirow[t]{2}{*}{ Insecticide } & \multicolumn{2}{|c|}{ Minepa $\left(8.271^{\circ} \mathrm{S}, 36.677^{\circ} \mathrm{E}\right)$} & \multicolumn{2}{|c|}{ Mavimba $\left(8.312^{\circ} \mathrm{S}, 36.677^{\circ} \mathrm{E}\right)$} & \multicolumn{2}{|c|}{ Lupiro (8.385 S, 36.670॰ E) } \\
\hline & & Dry season & Wet season & Dry season & Wet season & Dry season & Wet season \\
\hline \multirow[t]{10}{*}{ Female mosquitoes } & $0.75 \%$ permethrin a,b & $100^{5 S}$ & $100^{55}$ & $100^{55}$ & $100^{5 S}$ & $100^{5 S c}$ & $72.0^{R R c}$ \\
\hline & $0.05 \%$ deltamethrin & $86.0^{R R c}$ & $56.3^{R R c}$ & $87.0^{\mathrm{RR}}$ & $90.0^{\mathrm{RS}}$ & $8.0^{R R c}$ & $87.5^{R R c}$ \\
\hline & 0.05\% lambda-cyhalothrin & $60.0^{R R}$ & $82.5^{R R}$ & $76.3^{R R c}$ & $91.3^{\mathrm{RSC}}$ & $80.0^{\mathrm{RRC}}$ & $87.5^{R R C}$ \\
\hline & $4 \%$ dieldrin & $94.0^{\mathrm{RS}}$ & $98.8^{55}$ & $98.8^{\mathrm{SS}}$ & $100^{55}$ & $100^{55}$ & $100^{55}$ \\
\hline & $4 \%$ DDT & $92.0^{\mathrm{RS}}$ & $95.0^{\mathrm{RS}}$ & $87.5^{\mathrm{RR}}$ & $91.3^{\mathrm{RS}}$ & $78.0^{\mathrm{RR}}$ & $71.0^{\mathrm{RR}}$ \\
\hline & $0.1 \%$ propoxur & $94.0^{\mathrm{RS}}$ & $100^{5 S}$ & $91.3^{\mathrm{RSC}}$ & $100^{5 S}$ & $100^{5 S}$ & $98.0^{5 S}$ \\
\hline & $0.1 \%$ bendiocarb $b^{a, b}$ & $29.0^{R R c}$ & $99.0^{5 S c}$ & $98.0^{55}$ & $100^{5 S c}$ & $100^{5 S}$ & $99.0^{5 S}$ \\
\hline & $0.25 \%$ pirimiphos-methyl & $100^{55}$ & $100^{55}$ & $90.0^{\mathrm{RSC}}$ & $100^{5 S c}$ & $100^{S S}$ & $100^{5 S}$ \\
\hline & $5 \%$ malathion & $100^{5 S}$ & $100^{S S}$ & $100^{55}$ & $97.5^{55}$ & $99.0^{55}$ & $100^{5 S}$ \\
\hline & Control (untreated paper) & 4.6 & 2.4 & 2.2 & 1.8 & 1.2 & 2.9 \\
\hline \multirow[t]{10}{*}{ Male mosquitoes } & $0.75 \%$ permethrin & $100^{S S}$ & $100^{5 S}$ & $98.8^{5 S}$ & $100^{5 S}$ & $100^{S S}$ & $100^{S S}$ \\
\hline & $0.05 \%$ deltamethrin ${ }^{b}$ & $90.0^{\mathrm{RS}}$ & $93.0^{\mathrm{RS}}$ & $97.5^{\mathrm{RS}}$ & $98.8^{5 S}$ & $99.0^{5 S}$ & $95.0^{\mathrm{RS}}$ \\
\hline & 0.05\% lambda-cyhalothrin ${ }^{a, b}$ & $88.0^{R R c}$ & $99.0^{5 S c}$ & $100^{5 S}$ & $98.8^{5 S}$ & $71.0^{\mathrm{RR}}$ & $92.0^{\mathrm{RS}}$ \\
\hline & $4 \%$ dieldrin & $100^{5 S}$ & $100^{5 S}$ & $100^{5 S}$ & $100^{S S}$ & $100^{5 S}$ & $100^{5 S}$ \\
\hline & $4 \% D D D T^{a, b}$ & $97.0^{\mathrm{RS}}$ & $95.0^{\text {RS }}$ & $77.0^{\mathrm{RR}}$ & $98.8^{55}$ & $99.0^{55}$ & $100^{5 S}$ \\
\hline & $0.1 \%$ propoxur & $93.0^{\mathrm{RS}}$ & $99.0^{55}$ & $97.0^{\text {RS }}$ & $100^{5 S}$ & $100^{S S}$ & $100^{5 S}$ \\
\hline & $0.1 \%$ bendiocarb ${ }^{a, b}$ & $58.0^{R R c}$ & $100^{5 S c}$ & $100^{55}$ & $98.0^{5 S}$ & $100^{5 S}$ & $100^{5 S}$ \\
\hline & $0.25 \%$ pirimiphos-methyl & $100^{55}$ & $98.0^{55}$ & $97.0^{\mathrm{RS}}$ & $100^{5 S}$ & $100^{55}$ & $100^{5 S}$ \\
\hline & $5 \%$ malathion & $100^{5 S}$ & $100^{5 S}$ & $100^{55}$ & $100^{5 S}$ & $100^{5 S}$ & $100^{5 S}$ \\
\hline & Control (untreated paper) & 1.1 & 11.2 & 4.8 & 2.5 & 2.6 & 3.9 \\
\hline
\end{tabular}

Notes: Morphological identification of the Culex mosquitoes revealed $94 \%$ were $C x$. pipiens complex. Of these, PCR assays revealed that $81 \%$ were $C x$. quinquefasciatus, $2 \%$ were $C x$. pipiens pipiens and $3 \%$ were hybrids of the two species. About $14 \%$ of the specimens were non-amplified. These test results can therefore be considered primarily representative of $C x$. pipiens complex or more specifically for $C x$. quinquefasciatus

Abbreviations: SS, mosquitoes were susceptible to the test insecticide (WHO assays mortality between ( $98 \%$ and $100 \%$ ); RS, mosquitoes had reduced susceptibility indicating possible resistance and need for further investigation (mortality of 90-97\%); RR, mosquitoes were confirmed resistant to the test insecticide (WHO assays mortality below 90\%)

a Chemicals for which we observed differences in susceptibility of Culex mosquitoes between dry and wet seasons, i.e. where mosquitoes were fully susceptible in one season and fully resistant in a different season in same ward

${ }^{b}$ Chemicals for which we observed differences in susceptibility of Culex mosquitoes between (nearby) wards, i.e. where mosquitoes were fully susceptible in one ward and fully resistant in another ward during the same season

c There was a statistically significant difference in mortality between the dry and wet seasons

$\left.t_{(6)}=2.80, P=0.030\right)$. Similarly, pre-exposure to $4 \% \mathrm{PBO}$, followed by lambda-cyhalothrin increased mortality relative to exposure to lambda-cyhalothrin alone (66.3 vs $\left.28.8 \% ; t_{(6)}=6.60, P<0.001\right)$. There was a marginal increase in mortality when mosquitoes were pre-exposed to $20 \%$ TPP, followed by exposure to deltamethrin, compared to exposure to deltamethrin alone $(86.0 \mathrm{vs} 75.0 \%$; $\left.t_{(6)}=3.42, P=0.014\right)$. Pre-exposure to $4 \% \mathrm{PBO}$, followed by deltamethrin also resulted in higher mortality relative to cohorts exposed to deltamethrin only (60.0 vs $41.3 \%$; $\left.t_{(6)}=3.17 ; P=0.019\right)$. Lastly, pre-exposure to $20 \%$ DEM, followed by $4 \%$ DDT increased mortality in the synergized cohorts, compared to their un-synergized counterparts $\left(82.5\right.$ vs $\left.48.8 \% ; t_{(6)}=5.89, P=0.001\right)$.

\section{Estimated biting densities of Culex, relative to other mosquito species}

Of the 387,318 mosquitoes collected indoors during the sampling period, Culex constituted $77 \%(n=299,841)$ of the total catches. Of these, $79 \%$ were females $(n=236,484)$ and $21 \%$ males $(n=63,375)$. In total, 1053 Culex mosquitoes were subjected to species-specific identification; of these all were identified as members of the $C x$. pipiens complex (Table 6). 
Table 4 Mean \% mortality recorded 24 hours after exposure to lambda-cyhalothrin and deltamethrin, with and without synergist, TPP (triphenyl phosphate) or PBO (piperonyl butoxide). The mosquitoes tested were 3- to 5-day-old adult Culex mosquitoes reared from wild-collected larvae from Minepa and Mavimba wards in Ulanga District, Tanzania, in 2015 and 2016

\begin{tabular}{|c|c|c|c|c|c|c|}
\hline \multirow[t]{3}{*}{ Treatment } & \multirow{3}{*}{$\begin{array}{l}\text { No. of } \\
\text { replicates }\end{array}$} & \multirow[t]{3}{*}{ Sample size } & \multicolumn{4}{|c|}{ Mean \% mortality $(95 \% \mathrm{Cl})$} \\
\hline & & & \multicolumn{2}{|c|}{ Mavimba $\left(8.312^{\circ} \mathrm{S}, 36.677^{\circ} \mathrm{E}\right)$} & \multicolumn{2}{|c|}{ Minepa $\left(8.271^{\circ} \mathrm{S}, 36.677^{\circ} \mathrm{E}\right)$} \\
\hline & & & 0.05\% deltamethrin & $\begin{array}{l}\text { 0.05\% lambda- } \\
\text { cyhalothrin }\end{array}$ & $0.05 \%$ deltamethrin & 0.05\% lambda-cyhalothrin \\
\hline \multicolumn{7}{|c|}{ Tests with triphenyl phosphate (TPP) } \\
\hline Environmental control & 4 & 80 & 0 & $1.3(-2.7-5.2)$ & 0 & 0 \\
\hline Solvent control & 4 & 80 & 0 & 0 & 0 & 0 \\
\hline 20\% TPP only & 4 & 80 & 0 & $1.3(-2.7-5.2)$ & 0 & 0 \\
\hline $\begin{array}{l}\text { 20\% TPP and test insec- } \\
\text { ticide }\end{array}$ & 4 & 80 & $86.0(77.8-94.2)^{\mathrm{a}}$ & $83.8(76.1-91.4)^{\mathrm{a}}$ & $81.3(63.6-98.9)^{\mathrm{a}}$ & $73.8(48.3-99.2)^{\mathrm{a}}$ \\
\hline Test insecticide only & 4 & 80 & $75.0(68.9-81.1)^{b}$ & $72.5(62.2-82.8)^{b}$ & $51.3(23.7-79.8)^{b}$ & $71.3(47.5-95.2)^{\mathrm{a}}$ \\
\hline \multicolumn{7}{|c|}{ Tests with piperonyl butoxide (PBO) } \\
\hline Environmental control & 4 & 80 & 0 & 0 & 0 & 0 \\
\hline Solvent control & 4 & 80 & 0 & $1.3(-2.7-5.2)$ & 0 & 0 \\
\hline 4\% PBO only & 4 & 80 & 0 & $1.3(-2.7-5.2)$ & 0 & 0 \\
\hline $\begin{array}{l}\text { 4\% PBO and test Insec- } \\
\text { ticide }\end{array}$ & 4 & 80 & $60.0(42.8-77.2)^{\mathrm{a}}$ & $66.3(54.3-78.2)^{\mathrm{a}}$ & $93.8(86.1-101.4)^{\mathrm{a}}$ & $57.5(32.8-82.2)^{\mathrm{a}}$ \\
\hline Test insecticide only & 4 & 80 & $41.3(33.6-48.9)^{b}$ & $28.8(15.2-42.3)^{b}$ & $73.8(53.9-93.6)^{\mathrm{b}}$ & $35.0(20.5-49.5)^{b}$ \\
\hline
\end{tabular}

Notes: Morphological identification of the Culex mosquito populations revealed $94 \%$ were $C x$. pipiens complex. Of these, PCR assays revealed that $81 \%$ were $C x$. quinquefasciatus, $2 \%$ were $C x$. pipiens pipiens and $3 \%$ were hybrids of the two species. About $14 \%$ of the specimens were non-amplified. These test results can therefore be considered primarily representative of $C$ x. pipiens complex or more specifically for $C x$. quinquefasciatus. Environmental control refers to a control where mosquitoes are exposed to non-treated papers, and is used to assess any contamination in the test environment or during the procedures

$a, b$ The letters $a$ and $b$ signify statistically significant differences between \% mortalities obtained in tests with or without the synergists

Table 5 Mean \% mortality recorded 24 hours after exposure to 4\% DDT, with and without the synergist, diethyl maleate (DEM). The mosquitoes tested were 3- to 5-day-old adult Culex mosquitoes reared from wild collected larvae from Minepa and Mavimba wards in Ulanga District, Tanzania, in 2015 and 2016

\begin{tabular}{lllll}
\hline Treatment & No. of replicates & Sample size & & Mean \% mortality $(95 \% \mathrm{Cl})$ \\
\cline { 4 - 5 } & & & Mavimba $\left(8.312^{\circ} \mathrm{S}, 36.677^{\circ} \mathrm{E}\right)$ & Minepa $\left(8.271^{\circ} \mathrm{S}, 36.677^{\circ} \mathrm{E}\right)$ \\
\hline Environmental control & 4 & 80 & 0 & 0 \\
Solvent control & 4 & 80 & 0 & 0 \\
$20 \%$ DEM only & 4 & 80 & 0 & $90.0(83.5-96.5)^{\mathrm{a}}$ \\
$20 \%$ DEM and 4\% DDT & 4 & 80 & $82.5(67.3-97.7)^{\mathrm{a}}$ & $95.0(88.5-101.5)^{\mathrm{a}}$ \\
$4 \%$ DDT only & 4 & 80 & $48.8(38.7-58.8)^{\mathrm{b}}$ & 0
\end{tabular}

Notes: Morphological identification of the Culex mosquitoes revealed $94 \%$ were $C x$.pipiens complex. Of these, PCR assays revealed that $81 \%$ were $C x$. quinquefasciatus, $2 \%$ were $C x$. pipiens pipiens and $3 \%$ were hybrids of the two species. About $14 \%$ of the specimens were non-amplified. Environmental control refers to a control where mosquitoes are exposed to non-treated papers, and is used to assess any contamination in the test environment or during the procedures

$a, b$ The letters $a$ and $b$ signify statistically significant differences between \% mortalities obtained in tests with or without the synergists

\section{Discussion}

Until this study, the insecticide susceptibility status of non-malaria vectors such as Culex spp. was widely unknown in Tanzania, despite the abundance of these mosquito species. The present study investigated the susceptibility of $C x$. pipiens complex, to insecticides approved by the WHO for vector control. Standard WHO insecticide susceptibility bioassays were conducted separately for female and male Culex mosquitoes collected in different seasons and different wards.

Generally, Culex mosquitoes were found resistant to a wide range of pyrethroids, lambda-cyhalothrin and deltamethrin, DDT and the carbamate, bendiocarb. However, these species showed susceptibility to organophosphates, such as pirimiphos-methyl and malathion, 
Table 6 Relative abundance and indoor distribution of mosquitoes, across three study wards (including Minepa and Mavimba wards, from where Culex larvae were also obtained for the resistance tests). Data obtained from an annual mosquito surveillance programme conducted by the Ifakara Health Institute in Ulanga District, south-eastern Tanzania in 2012, 2013, and 2015

\begin{tabular}{|c|c|c|c|c|c|}
\hline Ward & Mosquito species & $\begin{array}{l}2012 \\
n(\%)\end{array}$ & $\begin{array}{l}2013 \\
n(\%)\end{array}$ & $\begin{array}{l}2015 \\
n(\%)\end{array}$ & Total \\
\hline \multirow[t]{5}{*}{ Minepa Ward } & Total mosquitoes collected & 57,393 & 23,448 & 39,359 & 120,200 \\
\hline & An. arabiensis, females ${ }^{\mathrm{a}}$ & $15,305(26.6)$ & $9224(39.3)$ & $10,950(27.8)$ & 35,479 \\
\hline & An. funestus group, females & $7713(13.4)$ & $1582(6.7)$ & $3097(7.9)$ & 12,392 \\
\hline & Cx.pipiens complex, males & $6469(11.2)$ & $2062(8.7)$ & $4160(10.5)$ & 12,691 \\
\hline & Cx. pipiens complex, females ${ }^{b}$ & $27,906(48.6)$ & $10,580(45.1)$ & $21,152(53.7)$ & 59,638 \\
\hline \multirow[t]{5}{*}{ Mavimba ward } & Total mosquitoes collected & 44,378 & 14,673 & 23,540 & 82,591 \\
\hline & An. arabiensis, females ${ }^{\mathrm{a}}$ & $4292(9.6)$ & $3158(21.5)$ & $2101(8.9)$ & 9551 \\
\hline & An. funestus group, females & $2460(5.5)$ & $894(6.0)$ & $793(3.4)$ & 4147 \\
\hline & Cx.pipiens complex, males & $8608(19.3)$ & $1418(9.6)$ & $3034(12.8)$ & 13,060 \\
\hline & Cx. pipiens complex, females ${ }^{b}$ & $29,018(65.4)$ & $9203(62.7)$ & $17,612(74.8)$ & 55,833 \\
\hline \multirow[t]{5}{*}{ Kivukoni ward } & Total mosquitoes collected & 98,902 & 34,374 & 51,251 & 184,527 \\
\hline & An. arabiensis, females ${ }^{\mathrm{a}}$ & $9572(9.6)$ & $4416(12.8)$ & $7070(13.7)$ & 21,058 \\
\hline & An. funestus group, females & $3327(3.3)$ & $663(1.9)$ & $860(1.6)$ & 4850 \\
\hline & Cx.pipiens complex, males & $18,905(19.1)$ & 7546 (21.9) & $11,155(21.7)$ & 37,606 \\
\hline & Cx. pipiens complex, females ${ }^{b}$ & $67,098(67.8)$ & $21,749(63.2)$ & $32,166(62.7)$ & 121,013 \\
\hline
\end{tabular}

a Sub-samples of An. gambiae complex mosquitoes collected in this area during this period have consistently been $100 \%$ An. arabiensis

b A sub-sample of 1053 Culex mosquitoes were subjected to further morphological examination and identified as Cx. pipiens complex

except for populations from the Mavimba ward, which were resistant to these insecticide classes.

While resistance was widespread across the study sites, lowest mortalities were observed against bendiocarb in Minepa (mortalities of $29 \%$ in female and $58 \%$ in male Culex mosquitoes) and against deltamethrin in Lupiro (8\% mortality in female Culex). Previous studies have reported that resistance in male Cx. pipiens and An. gambiae could potentially affect mating competitiveness in nature [50-52]. Additionally, information on insecticide susceptibility of male mosquitoes could be useful when designing interventions primarily against males, e.g. sterile insect technique (SIT) [53, 54], spraying of male swarms with insecticides [34], and use of attractive toxic sugar baits $[55,56]$.

In this part of south-eastern Tanzania, LLINs impregnated with permethrin remain the primary vector and diseases control interventions [35]. This study confirmed phenotypic resistance to this and also other public health insecticides that are currently not used in the study area. Our results suggest alternative sources of insecticide resistance selective pressure, most likely from agricultural pesticides [57-60]. Indeed, direct observation in the communities revealed an array of chemical classes widely sold and used for crop protection (Matowo et al., unpublished data). Therefore, for effective vector control, an integrated approach with agricultural pest control programmes in the allocation of insecticides is recommended. With reference to Culex spp., which also cause considerable biting nuisance in these communities where most people are small-holder farmers [37], the need for integrated pest and vector management and coordination among the public health and agriculture sectors is particularly important.

The significant differences in phenotypic resistance between the neighbouring wards, as revealed in this study, has also been reported for the malaria vector An. arabiensis [29], and clearly suggest that selection of insecticide resistance is happening at fine spatial scales and over time, e.g. between small administrative wards, other than variations previously reported between districts and regions [28, 61, 62]. The spatial and seasonal differences in insecticide resistance in $C x$. pipiens complex species could be influenced by the presence/use of various insecticides at a particular geographical area and time, even though this study did not directly assess the link between the two. These variations signify an important challenge for vector and disease control programmes when choosing insecticides for particular time periods and locations. For example, susceptibility was generally higher in the wet season than in the dry season. Thus, the possibility that insecticide-based interventions aimed at the wet season may have greater entomological and epidemiological impact on mosquito densities and disease transmission than those in the dry season, should be investigated. These fine-scale spatial and temporal 
variations are increasingly being reported across multiple sites. In one study in a single area in Mexico, both resistance phenotypes and genotypes were markedly varied at a fine spatial scale and time, in Aedes aegypti populations against chorpyrifos-ethyl and deltamethrin, driven by fine-scale pressure from the household insecticides use [63]. Similarly, Grossman et al. [64] recently reported spatial and seasonal heterogeneity in the frequency of $k d r$ haplotypes in Ae. aegypti from Mexico, likely to be influenced by differences in the usage of insecticides in space and time. In Uganda, there were monthly variations in $k d r$ allele frequency in Plasmodium-infected An. gambiae (s.s.) and the resistance was significantly higher in the dry compared to the wet season which is likely to be caused by seasonal changes in insecticide pressure [65]. A recent report by Jones et al. [22] on insecticide resistance in $C x$. quinquefasciatus from Zanzibar showed variability of resistance levels between nearby study sites, though the results were incomparable due to differences of Culex species at these sites. Niang et al. [66] also reported spatial variations of the L1014F $k d r$ allele found to dominate in An. arabiensis compared to An. coluzzii and An. gambiae sampled from 20 different study sites in the southeastern part of Senegal.

Mechanisms for resistance appear to be mixed. The partial suppression of pyrethroid resistance by synergist PBO and TPP exposures suggests that both P450 monooxygenases and esterases might be contributing to the pyrethroid resistance phenotypes observed in mosquito populations sampled from both Minepa and Mavimba wards. However, esterases seemed not to be involved in lambda-cyhalothrin resistance in mosquitoes from the Minepa ward, as only minimal change in mortality was observed upon pre-exposure to the synergist. In addition, DDT resistance was significantly restored after being exposed to DEM, suggesting a role for GSTs in DDT resistance in the Mavimba ward. However, DDT was not affected by DEM in Minepa samples, suggesting no role for metabolic resistance mechanisms here. Besides metabolic resistance, other resistance mechanisms, such as $k d r$ mutation, could play a role and further research is required to identify the mechanisms of resistance. These observations are consistent with previous studies on incomplete suppressions of pyrethroids and DDT resistances due to pre-exposure to synergists [22, $30,31]$. Nonetheless, the multiplicity of resistance mechanisms in these mosquito populations is a major concern and should be considered by control programmes.

In line with the WHO Global Vector Control Response strategy [18], it is important to integrate control of different arthropod vectors. In this area, where malaria is certainly the most important mosquito-borne disease, $79 \%$ of biting risk indoors was associated with $C x$. pipiens mosquitoes. Despite long-term use of the permethrinbased Olyset ${ }^{\circledR}$ nets, which are regularly distributed via the national government's mass distribution campaigns [35], Culex mosquitoes were fortunately found susceptible to permethrin, except in the Lupiro ward in the wet season (Table 3). Nonetheless, as resistance continues to spread, additional approaches, such as improved housing, larval source management, and indoor residual spraying (IRS) with non-pyrethroids and non-carbamates, may be considered as alternatives against both Culex and malaria vectors.

The most abundant Culex species in tropical and subtropical countries, including East Africa, belong to the $C x$. pipiens complex, which contains $C x$. quinquefasciatus, $C x$. pipiens pipiens, $C x$. pipiens torrentium and $C x$. pipiens molestus [3, 4]. From our findings, 94\% of Culex belonged to the $C x$. pipiens complex, of which $81 \%$ were verified by PCR as $C x$. quinquefasciatus, $2 \%$ as $C x$. pipiens pipiens and $3 \%$ as hybrids of $C x$. pipiens pipiens and Cx. quinquefasciatus. A small proportion (14\%) of samples were non-amplified, suggesting other Culex species for which there were no primers to distinguish. The presence of hybrids of $C x$. pipiens pipiens and $C x$. quinquefasciatus suggests that these species cross-mate in the wild. Cx. quinquefasciatus was previously documented through morphological identification as the dominant Culex species in the Kilombero Valley, where it occurred alongside a few $C x$. theileri and $C x$. univittatus [1]. However, none of these species were confirmed by PCR [1].

An important limitation of this study is that we analysed only a relatively small number of Culex to identify other possible Culex sibling species using PCR techniques due to the lack of appropriate primers. It should also be noted that synergists findings presented here stem from only female Culex specimens sampled in the dry season in Minepa and Mavimba wards as synergist papers are not easily accessible and not produced in bulk.

\section{Conclusions}

Culex pipiens complex, which mostly consists of $C x$. quinquefasciatus, are the most abundant Culex species in the study area, and contribute to more than $79 \%$ of all biting risk experienced in houses. The species are resistant to carbamates and pyrethroids commonly used in public health inside houses and also to DDT. The organophosphate, pirimiphos-methyl, which is also available for IRS however remains effective. This study has also demonstrated that insecticide resistance phenotypes and the underlying mechanisms varies considerably at fine geographical scales, suggesting some modifications to current insecticide resistance monitoring plans. Monooxygenases and esterases partly underlie the resistance phenotypes against pyrethroids, while GSTs play an 
important role in DDT resistance. Further investigations are required to identify more drivers and other mechanisms of resistance in Culex species across the wards. Overall, the extent of resistance reported in the current study indicates that additional approaches, such as improved housing, community-based larval source management, and IRS with non-pyrethroids and non-carbamates, should be considered as complementary vector control strategies. Lastly, resistances against insecticides not currently used for vector control in the villages, suggests possible linkages to agricultural pesticides use. Hence, multi-sectorial approaches should be encouraged to improve management of insecticide resistance.

\section{Acknowledgements}

We are grateful to the communities of Ulanga wards for giving us a permission to collect mosquito larvae from their farms. We highly acknowledge Paulina K. Kasanga and Neema K. Nombo for assisting with the mosquito larvae sampling and adults rearing. We appreciate the anonymous reviewers and editor for their initial detailed comments and suggestions, which have further improved the clarity and quality of this manuscript.

\section{Authors' contributions}

NSM and FOO conceived the study. NSM, EWK and FOO designed the experiments. NSM, SAM and EWK performed the field experiments under the mentorship of MT, LLK, MC, JU and FOO. GM facilitated the training on synergist tests and $\mathrm{DO}$ and $\mathrm{MC}$ supported in the morphological identifications of Culex species. NSM, SA and GM performed molecular analysis. NSM, GM, HSN and FOO analysed the data. NSM drafted the original manuscript. NSM, GM, MT, DO, LLK, MC, JU and FOO reviewed and revised the initial draft. All authors read and approved the final manuscript.

\section{Funding}

This work was financially supported by Wellcome Trust Masters Fellowship (grant no. WT104029/Z/14/Z) awarded to the lead author (NSM) and Wellcome Trust Intermediate Research Fellowship (grant no. WT102350/Z/13/Z) awarded to the senior author (FOO). NSM receives training support from Swiss Government Excellence Scholarship via the Federal Commission for Scholarships for Foreign Students FCS (ESKAS-Nr: 2017.0786), while FOO was supported by WHO/TDR (grant no. B40445). MC and LLK are supported by a DST/NRF SA Research Chair grant.

\section{Availability of data and materials}

The datasets used and/or analysed during the current study are available from the corresponding author upon reasonable request.

\section{Ethics approval and consent to participate}

Following a detailed introduction of the aims, study procedures, potential risks and benefits, written informed consent was obtained from all the owners of the farms where larvae sampling was carried out. This study received ethical review and approval from institutional review board of the Ifakara Health Institute (IHI; Ifakara, Tanzania; reference no. IHI/IRB/NO: 34-2014) and the Medical Research Coordinating Committee at the National Institute for Medical Research in Tanzania (reference no. NIMR/HQ/R.8a/Nol.IX/1903). This work is published upon the permission of the Director General of the National Institute for Medical Research in Tanzania (reference no. NIMR/HQ/P.12 VOL $X X V I I / 1)$.

\section{Competing interests}

The authors declare that they have no competing interests.

\section{Author details}

${ }^{1}$ Environmental Health and Ecological Sciences Department, Ifakara Health Institute, Ifakara, Tanzania. ${ }^{2}$ Swiss Tropical and Public Health Institute, Basel, Switzerland. ${ }^{3}$ University of Basel, Basel, Switzerland. ${ }^{4}$ Department of Disease Control, London School of Hygiene and Tropical Medicine, London, UK. ${ }^{5}$ Wits
Research Institute for Malaria, Wits/SAMRC Collaborating Centre for Multi-Disciplinary Research on Malaria, School of Pathology, Faculty of Health Sciences, University of the Witwatersrand, Johannesburg, South Africa. ${ }^{6}$ Centre for Emerging Zoonotic \& Parasitic Diseases, National Institute for Communicable Diseases, Johannesburg, South Africa. ${ }^{7}$ US Army Medical Research Directorate-Africa, Kisumu, Kenya. ${ }^{8}$ Institute of Biodiversity, Animal Health and Comparative Medicine, University of Glasgow, Glasgow, UK. ${ }^{9}$ School of Public Health, Faculty of Health Sciences, University of the Witwatersrand, Johannesburg, South Africa.

Received: 22 March 2019 Accepted: 19 August 2019

Published online: 23 August 2019

\section{References}

1. Ogoma SB, Lweitoijera DW, Ngonyani H, Furer B, Russell TL, Mukabana WR, et al. Screening mosquito house entry points as a potential method for integrated control of endophagic filariasis, arbovirus and malaria vectors. PLoS Negl Trop Dis. 2010;4:e773.

2. Hamon J, Burnett G, Adam J-P, Rickenbach A, Grjébine A. Culex pipiens fatigans Wiedemann, Wuchereria bancrofti Cobbold and the economic development of tropical Africa. Bull World Health Organ. 1967;37:217.

3. Subra R. Biology and control of Culex pipiens quinquefasciatus Say, 1823 (Diptera, Culicidae) with special reference to Africa. Int J Trop Insect Sci. 1981;1:319-38.

4. Mweya CN, Kimera SI, Mellau LS, Mboera LE. Inter-epidemic abundance and distribution of potential mosquito vectors for Rift Valley fever virus in Ngorongoro District, Tanzania. Glob Health Action. 2015;8:25929.

5. Bhattacharya S, Basu P, Sajal Bhattacharya C. The southern house mosquito, Culex quinquefasciatus: profile of a smart vector. J Entomol Zool Stud. 2016:4:73-81.

6. WHO. First WHO report on neglected tropical diseases: working to overcome the global impact of neglected tropical diseases. Geneva: World Health Organization; 2010.

7. CDC. Fast facts on neglected tropical diseases (NTDs). Atlanta: Centers for Disease Control and Prevention; 2015.

8. Turell MJ, Linthicum KJ, Patrican LA, Davies FG, Kairo A, Bailey CL. Vector competence of selected African mosquito (Diptera: Culicidae) species for Rift Valley fever virus. J Med Entomol. 2008:45:102-8.

9. Farajollahi A, Fonseca DM, Kramer LD, Kilpatrick AM. "Bird biting” mosquitoes and human disease: a review of the role of Culex pipiens complex mosquitoes in epidemiology. Infect Genet Evol. 2011;11:1577-85.

10. Weissenböck H, Hubálek Z, Bakonyi T, Nowotny N. Zoonotic mosquito-borne flaviviruses: worldwide presence of agents with proven pathogenicity and potential candidates of future emerging diseases. Vet Microbiol. 2010;140:271-80.

11. Pfeffer M, Dobler G. Emergence of zoonotic arboviruses by animal trade and migration. Parasit Vectors. 2010;3:35.

12. Brugman V, Hernández-Triana L, Medlock J, Fooks A, Carpenter S, Johnson $\mathrm{N}$. The role of Culex pipiens L. (Diptera: Culicidae) in virus transmission in Europe. Int J Environ Res Public Health. 2018;15:389.

13. Heinrich N, Saathoff E, Weller N, Clowes P, Kroidl I, Ntinginya E, et al. High seroprevalence of Rift Valley fever and evidence for endemic circulation in Mbeya region, Tanzania, in a cross-sectional study. PLoS Negl Trop Dis. 2012;6:e1557.

14. Mweya CN, Kimera SI, Kija JB, Mboera LE. Predicting distribution of Aedes aegypti and Culex pipiens complex, potential vectors of Rift Valley fever virus in relation to disease epidemics in East Africa. Infect Ecol Epidemiol. 2013;3:21748

15. Sang R, Kioko E, Lutomiah J, Warigia M, Ochieng C, O'Guinn M, et al. Rift Valley fever virus epidemic in Kenya, 2006/2007: the entomologic investigations. Am J Trop Med Hyg. 2010;83(Suppl. 2):28-37.

16. Hertz JT, Munishi OM, Ooi EE, Howe S, Lim WY, Chow A, et al. Chikungunya and dengue fever among hospitalized febrile patients in northern Tanzania. Am J Trop Med Hyg. 2012;86:171-7.

17. Chipwaza B, Mugasa JP, Selemani M, Amuri M, Mosha F, Ngatunga SD, Gwakisa PS. Dengue and chikungunya fever among viral diseases in outpatient febrile children in Kilosa district hospital, Tanzania. PLoS Negl Trop Dis. 2014;8:e3335. 
18. WHO. Global vector control response 2017-2030: a strategic approach to tackle vector-borne diseases. Geneva: World Health Organization; 2017.

19. WHO. Global plan for insecticide resistance management in malaria vectors. Geneva: World Health Organization; 2012.

20. Kelly-Hope L, Ranson H, Hemingway J. Lessons from the past: managing insecticide resistance in malaria control and eradication programmes. Lancet Infect Dis. 2008;8:387-9.

21. Curtis C, Pasteur N. Organophosphate resistance in vector populations of the complex of Culex pipiens L. (Diptera: Culicidae). Bull Entomol Res. 1981;71:153-61.

22. Jones CM, Machin C, Mohammed K, Majambere S, Ali AS, Khatib BO, et al. Insecticide resistance in Culex quinquefasciatus from Zanzibar: implications for vector control programmes. Parasit Vectors. 2012;5:78.

23. Pates H, Lines J, Keto A, Miller J. Personal protection against mosquitoes in Dar es Salaam, Tanzania, by using a kerosene oil lamp to vaporize transfluthrin. Med Vet Entomol. 2002;16:277-84.

24. Chavasse D, Lines J, Ichimori K, Marijani J. Mosquito control in Dar es Salaam. I. Assessment of Culex quinquefasciatus breeding sites prior to intervention. Med Vet Entomol. 1995;9:141-6.

25. Djègbè I, Boussari $O$, Sidick $A$, Martin T, Ranson H, Chandre F, Akogbéto $\mathrm{M}$, Corbel V. Dynamics of insecticide resistance in malaria vectors in Benin: first evidence of the presence of L1014S kdr mutation in Anopheles gambiae from West Africa. Malar J. 2011;10:261.

26. Orshan L, Kelbert M, Pener H. Patterns of insecticide resistance in larval Culex pipiens populations in Israel: dynamics and trends. J Vector Ecol. 2005;30:289.

27. Silvestrini F, Severini C, di Pardo V, Romi R, de Matthaeis E, Raymond M. Population structure and dynamics of insecticide resistance genes in Culex pipiens populations from Italy. Heredity. 1998;81:342.

28. Kabula B, Tungu P, Malima R, Rowland M, Minja J, Wililo R, et al. Distribution and spread of pyrethroid and DDT resistance among the Anopheles gambiae complex in Tanzania. Med Vet Entomol. 2014;28:244-52.

29. Matowo NS, Munhenga G, Tanner M, Coetzee M, Feringa WF, Ngowo $\mathrm{HS}$, et al. Fine-scale spatial and temporal heterogeneities in insecticide resistance profiles of the malaria vector, Anopheles arabiensis in rural south-eastern Tanzania. Wellcome Open Res. 2017;2:96.

30. Chouaïbou M, Zivanovic GB, Knox TB, Jamet HP, Bonfoh B. Synergist bioassays: a simple method for initial metabolic resistance investigation of field Anopheles gambiae s.l. populations. Acta Trop. 2013;130:108-11.

31. Nwane P, Etang J, Chouaïbou M, Toto JC, Koffi A, Mimpfoundi R, Simard F. Multiple insecticide resistance mechanisms in Anopheles gambiae s.l. populations from Cameroon, Central Africa. Parasit Vectors. 2013;6:41.

32. Jao LT, Casida JE. Insect pyrethroid-hydrolyzing esterases. Pestic Biochem Physiol. 1974;4:465-72.

33. Farnham AW. 12. The mode of action of piperonyl butoxide with reference to studying pesticide resistance. In: Farnham AW, editor. Piperonyl Butoxide. The Insecticide Synergist. Amsterdam: Academic Press; 1999. p. 199-213.

34. Diabate A, Tripet F. Targeting male mosquito mating behaviour for malaria control. Parasit Vectors. 2015;8:347.

35. Renggli S, Mandike R, Kramer K, Patrick F, Brown NJ, McElroy PD, et al. Design, implementation and evaluation of a national campaign to deliver 18 million free long-lasting insecticidal nets to uncovered sleeping spaces in Tanzania. Malar J. 2013;12:85

36. Kato F. Development of a major rice cultivation area in the Kilombero Valley, Tanzania. Japan: Afr Study Monogr. 2007;36(Suppl):3-18.

37. Swai JK, Finda MF, Madumla EP, Lingamba GF, Moshi IR, Rafiq MY, et al. Studies on mosquito biting risk among migratory rice farmers in rural south-eastern Tanzania and development of a portable mosquito-proof hut. Malar J. 2016;15:564.

38. Kaindoa EW, Matowo NS, Ngowo HS, Mkandawile G, Mmbando A, Finda M, Okumu FO. Interventions that effectively target Anopheles funestus mosquitoes could significantly improve control of persistent malaria transmission in south-eastern Tanzania. PLoS ONE. 2017:12:e0177807.

39. Okumu FO, Chipwaza B, Madumla EP, Mbeyela E, Lingamba G, Moore J, et al. Implications of bio-efficacy and persistence of insecticides when indoor residual spraying and long-lasting insecticide nets are combined for malaria prevention. Malar J. 2012;11:378.

40. Sattler MA, Mtasiwa D, Kiama M, Premji Z, Tanner M, Killeen GF, Lengeler C. Habitat characterization and spatial distribution of Anopheles sp. mosquito larvae in Dar es Salaam (Tanzania) during an extended dry period. Malar J. 2005;4:1.

41. WHO. Test procedures for insecticide resistance monitoring in malaria vector mosquitoes. Geneva: World Health Organisation; 2016.

42. Hunt R, Brooke B, Pillay C, Koekemoer L, Coetzee M. Laboratory selection for and characteristics of pyrethroid resistance in the malaria vector Anopheles funestus. Med Vet Entomol. 2005;19:271-5.

43. Kaindoa EW, Mkandawile GB, Lingamba GF, Killeen GF, Okumu FO. Longitudinal surveillance of disease-transmitting mosquitoes in rural Tanzania: creating an entomological framework for evaluation. Lancet. 2013;381:S70.

44. Kaindoa EW, Mkandawile G, Ligamba G, Kelly-Hope LA, Okumu FO. Correlations between household occupancy and malaria vector biting risk in rural Tanzanian villages: implications for high-resolution spatial targeting of control interventions. Malar J. 2016;15:199.

45. Lines J, Curtis C, Wilkes T, Njunwa K. Monitoring human-biting mosquitoes (Diptera: Culicidae) in Tanzania with light-traps hung beside mosquito nets. Bull Entomol Res. 1991;81:77-84.

46. Edwards FW. Mosquitoes of the Ethiopian Region. III. Culicine adults and pupae. London: British Museum Natural History; 1941. p. 1-515.

47. Smith $J$, Fonseca DM. Rapid assays for identification of members of the Culex (Culex) pipiens complex, their hybrids, and other sibling species (Diptera: Culicidae). Am J Trop Med Hyg. 2004;70:339-45.

48. Abbott W. A method of computing the effectiveness of an insecticide. J Econ Entomol. 1925;18:265-7.

49. R Core Team. R: A language and environment for statistical computing. Vienna: R Foundation for Statistical Computing; 2012

50. Berticat C, Boquien G, Raymond M, Chevillon C. Insecticide resistance genes induce a mating competition cost in Culex pipiens mosquitoes. Genet Res. 2002;79:41-7.

51. Platt N, Kwiatkowska R, Irving H, Diabaté A, Dabire R, Wondji C. Targetsite resistance mutations ( $k d r$ and $\mathrm{RDL}$ ), but not metabolic resistance, negatively impact male mating competiveness in the malaria vector Anopheles gambiae. Heredity. 2015;115:243-52.

52. Rowland M. Activity and mating competitiveness of $\mathrm{yHCH}$ /dieldrin resistant and susceptible male and virgin female Anopheles gambiae and An. stephensi mosquitoes, with assessment of an insecticide-rotation strategy. Med Vet Entomol. 1991;5:207-22.

53. Bellini R, Calvitti M, Medici A, Carrieri M, Celli G, Maini S. Use of the sterile insect technique against Aedes albopictus in Italy: first results of a pilot trial. In: Vreysen MJB, Robinson AS, Hendrichs J, editors. Area-wide control of insect pests. Dordrecht: Springer; 2007. p. 505-15.

54. Harris AF, McKemey AR, Nimmo D, Curtis Z, Black I, Morgan SA, et al. Successful suppression of a field mosquito population by sustained release of engineered male mosquitoes. Nat Biotechnol. 2012;30:828-30.

55. Müller GC, Beier JC, Traore SF, Toure MB, Traore MM, Bah S, et al. Successfu field trial of attractive toxic sugar bait (ATSB) plant-spraying methods against malaria vectors in the Anopheles gambiae complex in Mali, West Africa. Malar J. 2010:9:210.

56. Stewart ZP, Oxborough RM, Tungu PK, Kirby MJ, Rowland MW, Irish SR. Indoor application of attractive toxic sugar bait (ATSB) in combination with mosquito nets for control of pyrethroid-resistant mosquitoes. PLoS ONE. 2013:8:e84168.

57. Riaz MA, Poupardin R, Reynaud S, Strode C, Ranson H, David J-P. Impact of glyphosate and benzo pyrene on the tolerance of mosquito larvae to chemical insecticides. Role of detoxification genes in response to xenobiotics. Aquat Toxicol. 2009;93:61-9.

58. Poupardin R, Reynaud S, Strode C, Ranson H, Vontas J, David J-P. Crossinduction of detoxification genes by environmental xenobiotics and insecticides in the mosquito Aedes aegypti: impact on larval tolerance to chemical insecticides. Insect Biochem Mol Biol. 2008;38:540-51.

59. David J-P, Coissac E, Melodelima C, Poupardin R, Riaz MA, Chandor-Proust A, Reynaud S. Transcriptome response to pollutants and insecticides in the dengue vector Aedes aegypti using next-generation sequencing technology. BMC Genomics. 2010;11:216.

60. Nkya TE, Akhouayri I, Kisinza W, David J-P. Impact of environment on mosquito response to pyrethroid insecticides: facts, evidences and prospects. Insect Biochem Mol Biol. 2013;43:407-16.

61. Kisinza WKB, Tungu P, Sindato C, Mweya C, Massue D, Emidi B, et al. Detection and monitoring of insecticide resistance in malaria vectors in 
Tanzania Mainland. Tanzania: Technical Report of the National Institute for Medical Research; 2011.

62. Ranson H, N'Guessan R, Lines J, Moiroux N, Nkuni Z, Corbel V. Pyrethroid resistance in African anopheline mosquitoes: what are the implications for malaria control? Trends Parasitol. 2011;27:91-8.

63. Deming R, Manrique-Saide P, Barreiro AM, Cardeña EUK, Che-Mendoza $A$, Jones $B$, et al. Spatial variation of insecticide resistance in the dengue vector Aedes aegypti presents unique vector control challenges. Parasit Vectors. 2016:9:67.

64. Grossman MK, Rodriguez J, Barreiro AM, Lenhart A, Manrique-Saide P, Vazquez-Prokopec GM. Fine-scale spatial and temporal dynamics of $\mathrm{kdr}$ haplotypes in Aedes aegypti from Mexico. Parasit Vectors. 2019;12:20.

65. Verhaeghen K, Van Bortel W, Roelants P, Okello PE, Talisuna A, Coosemans M. Spatio-temporal patterns in kdr frequency in permethrin and
DDT resistant Anopheles gambiae s.s. from Uganda. Am J Trop Med Hyg. 2010;82:566-73.

66. Niang EHA, Konaté L, Diallo M, Faye O, Dia I. Patterns of insecticide resistance and knock down resistance (kdr) in malaria vectors An. arabiensis, An. coluzzii and An. gambiae from sympatric areas in Senegal. Parasit Vectors. 2016;9:71.

\section{Publisher's Note}

Springer Nature remains neutral with regard to jurisdictional claims in published maps and institutional affiliations.
Ready to submit your research? Choose BMC and benefit from:

- fast, convenient online submission

- thorough peer review by experienced researchers in your field

- rapid publication on acceptance

- support for research data, including large and complex data types

- gold Open Access which fosters wider collaboration and increased citations

- maximum visibility for your research: over $100 \mathrm{M}$ website views per year

At BMC, research is always in progress.

Learn more biomedcentral.com/submissions 